\title{
Using Modern Technologies in the Production of Mining Equipment
}

\author{
Alexandr Korotkov ${ }^{1, *}$, Lidya Korotkova ${ }^{1}$, and Denis Vidin ${ }^{1}$ \\ ${ }^{1}$ T.F. Gorbachev Kuzbass State Technical University, Chair of Metal-Cutting Machines and Tools, \\ 65000028 Vesennyaya st., Kemerovo, Russian Federation
}

\begin{abstract}
After a period of operation imported mining equipment starts failing. As a rule, breakages and critical wear of parts do not involve the entire product, but its individual parts. Despite this, manufacturers of this equipment do not provide repair kits, but offer to change the units and assemblies entirely. As a result, the repair and maintenance of imported equipment imposes significant financial costs. This paper proposes ways to eliminate or reduce the additional costs. The problem solution is based on the determination of the chemical composition and structure of worn out or out-of-order parts, reproduction of their manufacturing technology and production of analogues of these parts in single quantities. The work is performed on the example of high-pressure pump parts and large-sized gears used in mining equipment. For high-pressure pumps, analogues of end sealing gaskets and bearing bushings were made. The obtained experience can be used in the manufacturing and restoration of mining equipment in the Kuzbass enterprises, which allows reducing financial costs and implementing import substitution. The research part of the work was performed in the metallurgical laboratories of KuzSTU, and the reproduction of worn out and out-of-order parts was carried out at enterprises which requested this study.
\end{abstract}

\section{Introduction}

The problem of operating imported units and assemblies, their replacement and maintainability is becoming increasingly urgent. This is due to the fact that most foreign manufacturers offer a complete replacement of units and assemblies where only a few parts can be defective. In such cases, repair kits are not provided to replace individual parts in assemblies and units. Since many Russian enterprises use imported equipment that does not have domestically produced counterparts, this problem leads to a significant increase in financial expenses of operation.

To solve this problem, technologies of manufacturing parts for mining equipment have been studied with the aim of their possible restoration or production. The analysis is made on the example of gear pump parts, as well as large-sized gears. For this purpose, on the basis of the Chair of Metal-Cutting Machines and Tools of KuzSTU, investigations on identification of materials from which the above parts are made have been carried out.

\footnotetext{
* Corresponding author: korotkov.a.n@,mail.ru
} 
Technologies of manufacturing these parts in domestic conditions are developed. The recommendations on manufacturing analogous parts are formulated or the technologies for restoring worn parts are proposed.

This approach allows significant reduction in the costs of repair work and provision of further maintenance for the units of imported mining equipment.

\section{Research methods}

In the course of research in this work, quality control of parts was carried out according to the developed method [1]. The methods used were based on existing state standards and included the following main stages:

1) visual inspection;

2) measurement of linear dimensions and configuration;

3) analysis of the chemical composition conducted to determine the qualitative and quantitative content of chemical elements in metal products with optical emission analysis;

4) determination of mechanical properties, by measuring the hardness of the working surfaces of the tested materials by the Rockwell method (scales B and C, GOST 9013);

5) analysis of macro and microstructure, by controlling the macrostructure with visual inspection and comparing it with the standards of scales in accordance with GOST 10243. Control of the steel microstructure is an important part of the quality assessment. The list of controlled parameters and their acceptable values are regulated in the technical conditions of standards for steel (GOST 5639, GOST 1763, GOST 1778, GOST 8233, GOST 5640).

The choice of controlled parameters is determined by the standards for the corresponding grades and types of materials produced domestically. If necessary, special research methods for specific products were developed. They are described further in the results and discussion section.

\section{Results and discussion}

The results of investigation in assemblies and parts of foreign production used in the mining industry are discussed below.

\subsection{Imported high-pressure pump}

At the coal mining enterprises of Kuzbass problems with high pressure gear pumps produced by Kracht are regular occurrence. In these pumps, used for pumping aggressive media, such details as end sealing gaskets, friction bearings bushing and shafts of gears wear intensively. As a result of the failure of these parts, a large number of defective pumps accumulate in the enterprises. Replacing them entirely requires considerable spending.

Studies showed that the gear wheel of the pump is made of high-carbon steel of grade 100Cr6 (according to DIN 1.3505) [2], which can be analogous to Russian steel grades of type X (GOST 5950) and ShKh15 (GOST 801) [3]. The gear is thermally treated for a maximum hardness of 60-64 HRC, and its microstructure consists of martensite and inclusions of secondary carbides. The carbides are evenly distributed and have a finely dispersed structure with a size of $1-2 \mu \mathrm{m}$, which is typical for steels manufactured by the powder metallurgy method $[4,5]$. On the contour, the surface of the gears is roller coated with a layer of fine-grained ferrite steel. This surface layer thickness is $0.08-0.10 \mathrm{~mm}$ [6].

An analysis of the microstructure of the gear-shaft materials made it possible to draw a conclusion about the features of its production technology. In particular, the gear was pressed onto the shaft, and then the working part of the shaft and the gear itself were 
subjected to quenching and low tempering, presumably with heating by high-frequency currents [7-9]. The roller coating of a thin layer of low-carbon ferrite steel along the perimeter of the gear is the final operation of manufacturing the gear shaft. [10]

The peculiarity of manufacturing of end sealings was studied. They are made of carbonless ferrite steel and a layer of tin-bronze powder is applied to their surface using powder metallurgy technology. The powder for the gaskets is applied by spraying. This was established by studying the macrostructure with a Brinell magnifier with an increase of $25 \times$. Analysis of the chemical composition of the sprayed layer shows that the chemical composition of this layer is similar to that of bronze: for a large gasket - GCuPb10Sn; for a small gasket - GCuPb22Sn (according to DIN 1716). An analogue of these bronzes is domestically produced tin bronzes of grades BrO8S12 (for a large gasket) and BrO5S25 (for a small gasket) in accordance with GOST 613.

Studies of the friction bearing bushing showed that it was made of a composite polymer material. Visual inspection of the bushing shows that the product has a metallic luster, without any traces of machining. The bushing surface has a relief characteristic of polymer products obtained by injection molding. Based on the studies conducted, it can be concluded that the bushing is manufactured with PIM technology (Powder Injection Molding). Such a technology, most commonly implemented as injection molding, is used for the mass production of complex and precise products from metal, ceramic or intermetallic powders of micron, submicron and nanosizes. It combines traditional plastic injection molding and powder sintering technology, providing details of complex geometric shapes in reusable metal tools and reducing or completely eliminating the need for further machining. [11-13]

\subsection{Large gears}

Repairing large parts such as shafts, gears, strikers and others presents a serious problem in the operation of mining equipment (conveyors, stone crushing machines, mining combine machines, etc.). The experience shows that when replacing the original parts with analog components made from steels similar in composition and hardened by traditional technology, their resistance is reduced by an order in magnitude.

At one of the Kuzbass enterprises, for the purpose of repair, a large-sized cogwheel $(\mathrm{Z}=$ $7, \mathrm{D}=370 \mathrm{~mm}, \mathrm{~h}=80 \mathrm{~mm}$ ) made of steel 20XH3A (according to GOST 4543) and $18 \mathrm{CrNi3}$ (DIN FRG) was manufactured and thermally processed with classical technology, recommended for this group of steels. The investigated steels belong to the group of highstrength chromium-nickel steels. By their typical mode of heat treatment they belong to carburizing steels. Engineering chromium-nickel steels belong to steels of deep hardenability, as they are quenched for the bainite structure in air.

As a method of surface hardening, the manufacturer used chemical-thermal treatment nitro-carburazing followed by complete and incomplete quenching, intermediate high tempering between quenching and final low tempering [14]. After such a classical treatment, the analogue part did not receive the required hardness and dramatically deteriorated during several days of operation.

To determine the reason for the high resistance of the original part, the working part of the tooth segment was examined.

In the course of macrostudy, it was established that a gear tooth has bimetallic structure. At the macrosection on the working part of the gear tooth segment, a forged insert was found (no traces of the dendritic macrostructure were found), resembling a trapezoidal geometric figure. In this case, the tooth body has the structure of the cast billet as some traces of the dendritic macrostructure were revealed on the macrosection. The chemical 
composition of the forged insert and cast tooth body is identical. The tooth body has an average hardness of 42-43 HRC; that of insert is 23-25 HRC.

The results of measuring the microhardness along the cross-section of the insert and the tooth body showed homogeneity of the structure along the cross-section with insignificant deviations within the limits of measurement accuracy. The microhardness in the tooth insert (the working part of the tooth) was uniform and amounted to 220-260 HV, which corresponds to the microstructure of sorbitol. The microhardness of the cast part of the tooth is less uniform and was mainly in the range 400-480 HV, with deviations down to $360 \mathrm{HV}$. More significant fluctuations of microhardness in the tooth body are explained by the fact that in the microstructure, along with bainite, soft inclusions of ferrite $(80 \mathrm{HV})$ are present. [15]

From the presented results of microstructure examinations and microhardness measurements it is established that the forged metal zone passes into the cast zone smoothly. Thus, there is no sharp transition between the forged insert and the cast body of the tooth. Owing to this production technology, the process of joining two bodies with close chemical composition is realized through heat bonding the insert metal. This can be done with the use of centrifugal casting. [16]

With centrifugal casting, the rate of crystallization can be controlled by the speed of rotation of the mold and the volume of the metal fed into the crystallization zone. In the case under consideration, a layered macrostructure with a layer thickness of about 20-40 $\mu \mathrm{m}$ is evidence of a thin-walled filling with centrifugal casting. Due to the high cooling rate during the centrifugal casting of the gear body, it is possible to harden the bainite structure from the liquid state during the crystallization process. This technology of producing billet allows excluding the quenching technological operation in the manufacture of gears. The final operation in the production of cast billets should be tempering or annealing to relieve stress.

Investigations of the microstructure and measurement of microhardness in the surface layers showed no presence of a nitro-carburizing or carburizing layer. However, microareas were found on the surface of the tooth insert with microhardness of up to HV 800 $\mathrm{kgf} / \mathrm{mm}^{2}$ at a depth of no more than $0.02 \mathrm{~mm}$. Probably, the cause of this increase in microhardness is hardening, or the remainder of the worn-out nitrided layer (the zone of a white non-friction surface layer).

Thus, the bimetallic design of the large-sized gear wheel (with forged insert in the working area of the wheel tooth) provides a number of advantages:

1) dispersed microstructure of the cast metal, providing increased strength of the wheel body;

2) the presence of a smooth transition zone from the insert to the gear body, which ensures the strength of their connection; body.

3) simplification of heat treatment by combining the casting and hardening of the wheel

The use of modern technologies in the production of large-sized products can significantly improve the microstructure and, as a result, improve durability and reduce the process of hardening heat treatment. The latter is one of the topical problems in the production of large-sized parts.

\section{Conclusion}

As the research has shown, modern technologies for manufacturing materials, billets and finished products, as well as modern methods for their processing are used in the production of imported mining equipment: powder metallurgy, centrifugal casting, surfacing, spraying, hardening by high-frequency currents and other methods. 
Each of the existing technologies has both advantages and disadvantages and should be used, based on ensuring the required operating parameters of the finished product. The units and assemblies of mining equipment are subject to high standards for reliability and durability to ensure their trouble-free operation.

The accumulated experience can be used in the domestic production of mining equipment, which will make its import substitution possible.

\section{References}

1. A.N. Korotkov, L.P. Korotkova, D.V. Vidin, E3S Web of Conferences, 21, 01008 (2017)

2. C. Wegst, M. Wegst, Stahlschlüssel-Taschenbuch (Verlag Stahlschluessel Wegst GmbH, Marbach, 2015)

4. B. Bramfitt, Metallographer's Guide: Practices and Procedures for Irons and Steels (ASM International, Manchester, 2002)

5. E. Berghof-Hasselbächer, Atlas of Microstructures (DGRT, Berlin, 2013)

6. H. Hiorns, Metallography: An Introduction to the Study of the Structure of Metals, Chiefly by the Aid of the Microscope (DGRT, Berlin, 2009)

7. G. Zlateva, Microstructure of Metals and Alloys: An Atlas of Transmission Electron Microscopy Images (Taylor\&Francis Group, London, 2008)

8. M. Shtremel, MiTOM. 8, 12 (1980)

10. M. Tuttle, Structural Analysis of Polymeric Composite Materials (Hardcover Book, Hannover, 2007)

11. Y. Hezhou, L. X. Yang, Journal of materials processing technology, 7, 12 (2008)

12. B. A. Mathew, R. Mastromatteo, Metal Powder Report, 57, 20 (2002)

13. B. Leszczyńska-Madej, M. Madej, Archives of Metallurgy and Materials, 3, 805 (2011)

14. A. Kvasova, B. Gerike, E. Murko, D. SkudarnovE3S Web of Conferences 21, 03016 (2017)

15. A. Zeren, Materials and Design, 28, 2344 (2007)

16. J. W. Gao, C. Y. Wang, Materials Science and Engineering, 2, 207 (2000) 